\title{
Modelling and experimental investigation of an active damper
}

\author{
Rafael Luís Teixeira*, Francisco Paulo Lépore Neto and José Francisco Ribeiro \\ College of Mechanical Engineering, Federal University of Uberlândia, Campus Santa Mônica, 38400-089, \\ Uberlândia-MG, Brazil
}

\begin{abstract}
This paper presents a validation methodology of the dynamic behavior of an active viscous damper. The damper has two flexible metallic bellows connected to a rigid reservoir filled with fluid. When one of the bellows is connected to a vibrating structure a periodic flow passes through a variable internal orifice and the damping effect is produced. The size of the orifice is adjusted by a controlled linear piezoelectric actuator that positions the conical core into a conical cavity. The device finite element structural model consists of the valve body and its conical core that are assumed rigid and the flexible bellows are represented by two pistons with elastic suspensions. The flow developed inside the damper is modeled considering the fluid-structure interation, using the Lagrangean-Eulerian formulation. To validate the proposed model a prototype was constructed and experimental tests and numerical simulations are accomplished in the time domain, applying harmonic excitations. The results are compared using curves that relate the damping coefficient with the orifice size and with the input velocity applied at the bellows face. However, for the proper control design and system operation, the direct use of the finite element model becomes unviable due to its high computational time. Then, a reduced second order discrete dynamic model for the damper was developed. The model parameters are identified by analysis in the frequency domain, using impulsive excitation force, for constant and variable orifice sizes. At low excitation frequencies, the damper prototype behaves like a single degree of freedom system which damping factor changes with the orifice size A fuzzy controller was designed and it generates the orifice reference size associated to the desired damping factor. The active system presented better performance when compared to the passive one.
\end{abstract}

Keywords: Active damper, finite element, fluid-structure interaction, fuzzy controller

\section{Introduction}

In several industrial applications it is necessary to absorb structural vibration energy, mainly when impulsive forces without any repetition pattern excites the mechanical system. In these cases, passive damping devices have low efficiency, because they are only designed for a specific operating condition. Frequently, hydraulic piston actuators are used when large damping forces are required. However, the implementation, operation and maintenance of this type of solution are very expensive. Commercial passive dampers used on regular vehicles are designed for a specific behavior and don't allow damping factor adjustments as function of the pavement type, even for comfort or stability operating conditions.

New conceptions of active dampers were proposed, investigated and tested in the last years. Giliomee et al. [2], Hagopian et al. [3] and Kitching et al. [5] developed dampers systems based on hydraulic piston whose variable orifice is controlled by an electrodynamic valve. Oh and Onoda [4] used metallic flexible bellows and magneto-rheologic fluids.

The proposed active damper consists in a two systems: an hydraulic-mechanical system that generates the damping force and a control system, which has a piezoelectric actuator to control the valve opening and closures. The hydraulic circuit has two metallic flexible bellows and a valve with variable orifice. The bellows is flexible in the

\footnotetext{
${ }^{*}$ Corresponding author. E-mail: rafael@mecanica.ufu.br.
} 


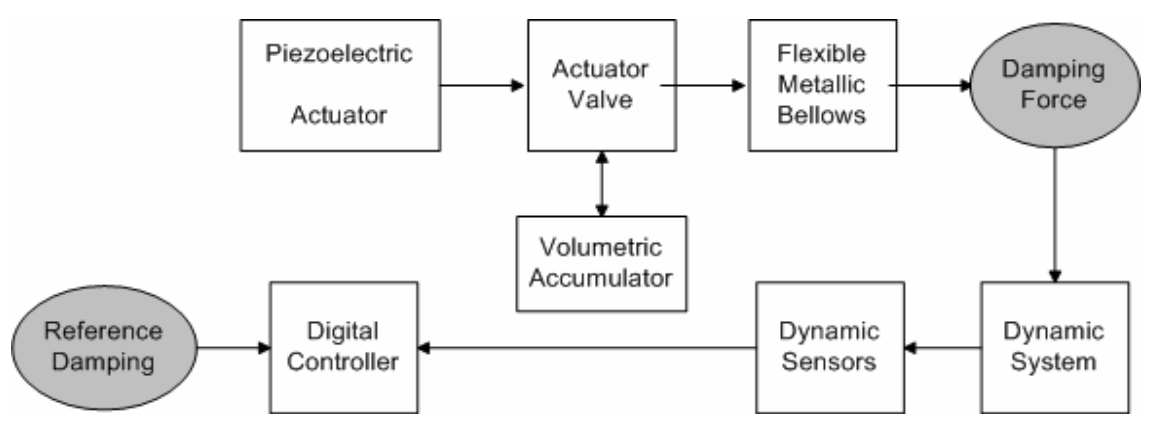

Fig. 1. Block Diagram of the proposed active damper.

axial direction and its extremities are connected at two points of the vibrating structure. The vibration motion, applied in the bellows axial direction, pumps the internal fluid through the valve orifice. The hydraulic circuit contains a volumetric accumulator, a variable orifice, and a metallic cylindrical bellows. The bellows is flexible in the axial direction and its extremities are connected at two points of the vibrating structure. The vibrating motion, applied in the bellows axial direction, pumps the internal fluid through the valve orifice. The displaced fluid volume travels between the bellows and the accumulator. The flow restriction caused by the valve orifice size result in pressure gradients at both side of hydraulic circuit. This internal pressure applied on the transversal area of the bellows generates the damping force that acts on the vibrating structure. The system becomes active because the valve closure can be adjusted by a position control system that uses a linear piezoelectric actuator.

The main objective of the work is to incorporate variable damping to the mechanical system, associated to each specific position of the valve; that is, a new absorber can be set at each sampling time of the state variables. This sampling rate depends on the actuator time response, on the external excitations and on the desired dynamic performance of the vibratory system.

In the block diagram shown in Fig. 1, it is assumed that the flexible metallic bellows is attached to a dynamic vibratory system and all state variables are measurable by appropriate sensors. Then, considering one reference damping coefficient and the associated system state, a digital controller will command the piezoelectric actuator to position the valve core. The bellows expansion and contraction movements produce pressure variations inside the hydraulic circuit. The internal pressure in the bellows acting on its cross section area results the value of the damping force, which depends on the control action applied by the piezoelectric actuator. To avoid the bellows structural collapse, the closure of the valve must be limited so that the internal pressure is always lesser than the maximum allowable pressure.

The efficiency of this device depends on the fluid viscosity and on the absence of mixed bubbles of gas dissolved in the fluid. To solve this problem a parallel circuit with a vacuum pump can provide a preliminary removal of the gas bubbles that are present in the working fluid. The choice of a suitable value of the static nominal pressure in the hydraulic circuit can prevent the dissolved gas bubble generation in the low-pressure region of the circuit.

Some initial parameters were specified for the design and construction of the active damper prototype. A commercial metallic bellows was specified. It supports internal pressures up to $3.8 \mathrm{MPa}$, has an effective cross section area of $383 \mathrm{~mm}^{2}$, resulting allowed axial loads up to $1300 \mathrm{~N}$. The bellows axial stroke is in range of $-8 \mathrm{~mm}$ up to $6 \mathrm{~mm}$, referenced to its undeformed state.

The piezoelectric actuator has an elliptical elastic structure with a pack of PZT4 elements installed at the larger semi-axis. The device output is mechanically amplified at the other semi-axis resulting a displacements range equal to $0.5 \mathrm{~mm}$ and a load capacity up to $570 \mathrm{~N}$, depending on the voltage applied to the piezoelectric elements.

The following sections will present the development of the finite element model of the damper device including the fluid-structure interaction, the design of the active damper prototype and the time domain experiments used to validate the finite element model. The active damper prototype is characterized in the frequency domain and a second order dynamic model is adjusted to represent its reduced model. Finally, numeric simulations are done with a passive (fixed orifice) and active (variable orifice) system. For the active system a fuzzy controller was designed. 


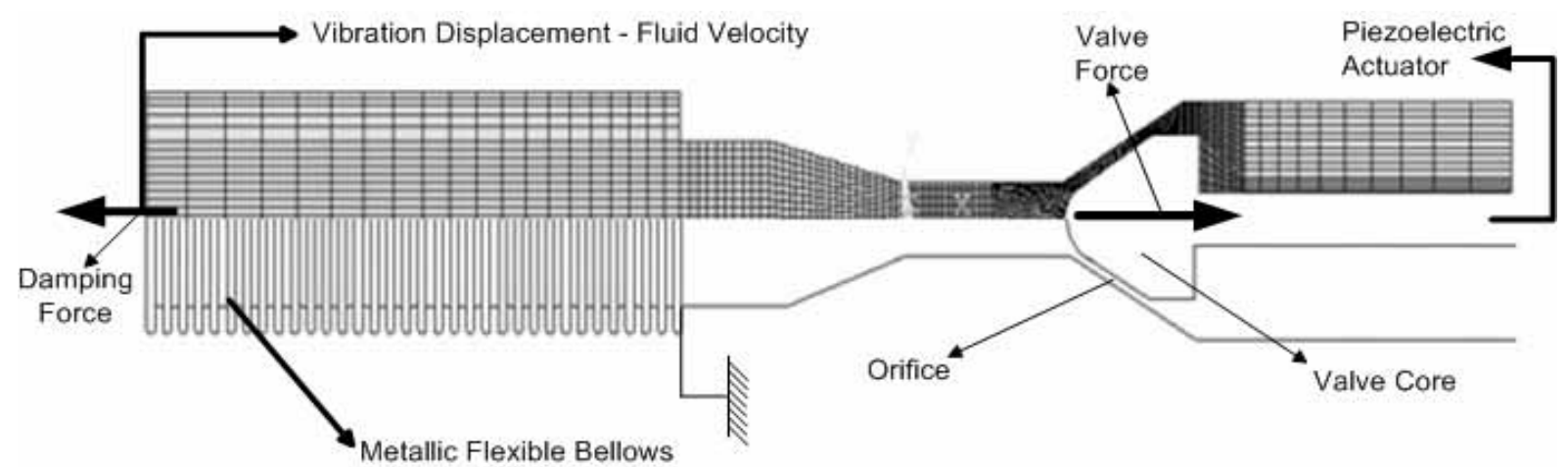

Fig. 2. Finite element axi-symmetric model of the damper device hydraulic circuit.

\section{Damper device finite element model}

The model of hydraulic circuit of the damper device is developed by the finite element technique. As shown in Fig. 2, the vibration applied at the left end of the bellows imposes a velocity to the fluid. At the right end of the hydraulic circuit a constant pressure is imposed. The valve orifice has conic shape. The orifice size can be adjusted by moving the conical core in the axial direction. In this region the fluid will reach higher velocities. When the bellows contracts the pressure increases at the region located at the left side of the core and is reduced at its right side. If the bellows expands, the pressure distribution will be reversed. The finite element model considers the valve body and the conical core as rigid structures. Each bellows is represented by rigid piston, with equivalent mass of its moving part, with an elastic suspension. The fluid-structure interaction occurs at the pistons faces which impose displacement to the internal fluid.

This model is used to determine the velocity and pressure fields developed in the fluid. Integrating the pressure on the core surface the force acting on the valve core is determined. Applying the same procedure on the end surface of the bellows, the effective damping force acting on the vibrating structure can be calculated. The computational model of the hydraulic circuit was developed using the module FLOTRAN CFD - Computational Fluid Dynamics ANSYS ${ }^{\circledR}$, considering the $\mathrm{K}-\varepsilon$ model of turbulence, developed Launder and Spalding [6]. According to the geometric characteristics of the problem, an axi-symmetric finite element model was designed. Figure 2 shows the configuration of the system components and the mesh geometry.

The model mesh shown by Fig. 3 has 2741 elements, is refined at the central region around the valve core, where higher velocity gradients occur. In this figure, the axial position of the conical core relative to the valve body is determined by the GAP variable measured at the X-axis of the inertial reference frame. Geometrically, GAP is the distance between the vertices of the triangles $\triangle \mathrm{abc}$ and $\triangle \mathrm{ABC}$. The spherical tip of the core is generated by the circumference inscribed in the triangle $\Delta \mathrm{abc}$. The right end of the core has a cylindrical shape, where the actuator rod is fixed. If the reference frames XY and xy are coincident GAP results equal to zero and the valve is completely closed.

The working fluid is a Newtonian lubricant oil with density equal to $7.70 \times 10^{2} \mathrm{~kg} / \mathrm{m}^{3}$ and viscosity equal to $0.0028 \mathrm{~kg} / \mathrm{ms}$. The applied boundary conditions are $V x=0$ and $V y=0$ indicating null velocities at all internal structure walls. Due to the model axi-symmetry $V x=0$ at the points where $X=0$.

The model of Fig. 2, without the end pistons, was used by Teixeira et al. [7] to calculate the fluid velocities and pressures distributions at the fluid domain in the steady state regime. Different values of positive and negative input velocity were imposed at left bellows face, using sampled values of the GAP range. Integrating the pressure over the core surface and on the bellows end face, the force at the core and the damping force were calculated, generating surfaces that map these forces in function of the velocity and the GAP.

The complete model, including the two pistons and the rigid structure that contains the fluid is shown by Fig. 4 . The transient state computational simulations were done by imposing a $3 \mathrm{~mm}$ amplitude sinusoidal displacement on the left bellows equivalent mass. The transient pressure and velocity distributions were calculated using different 


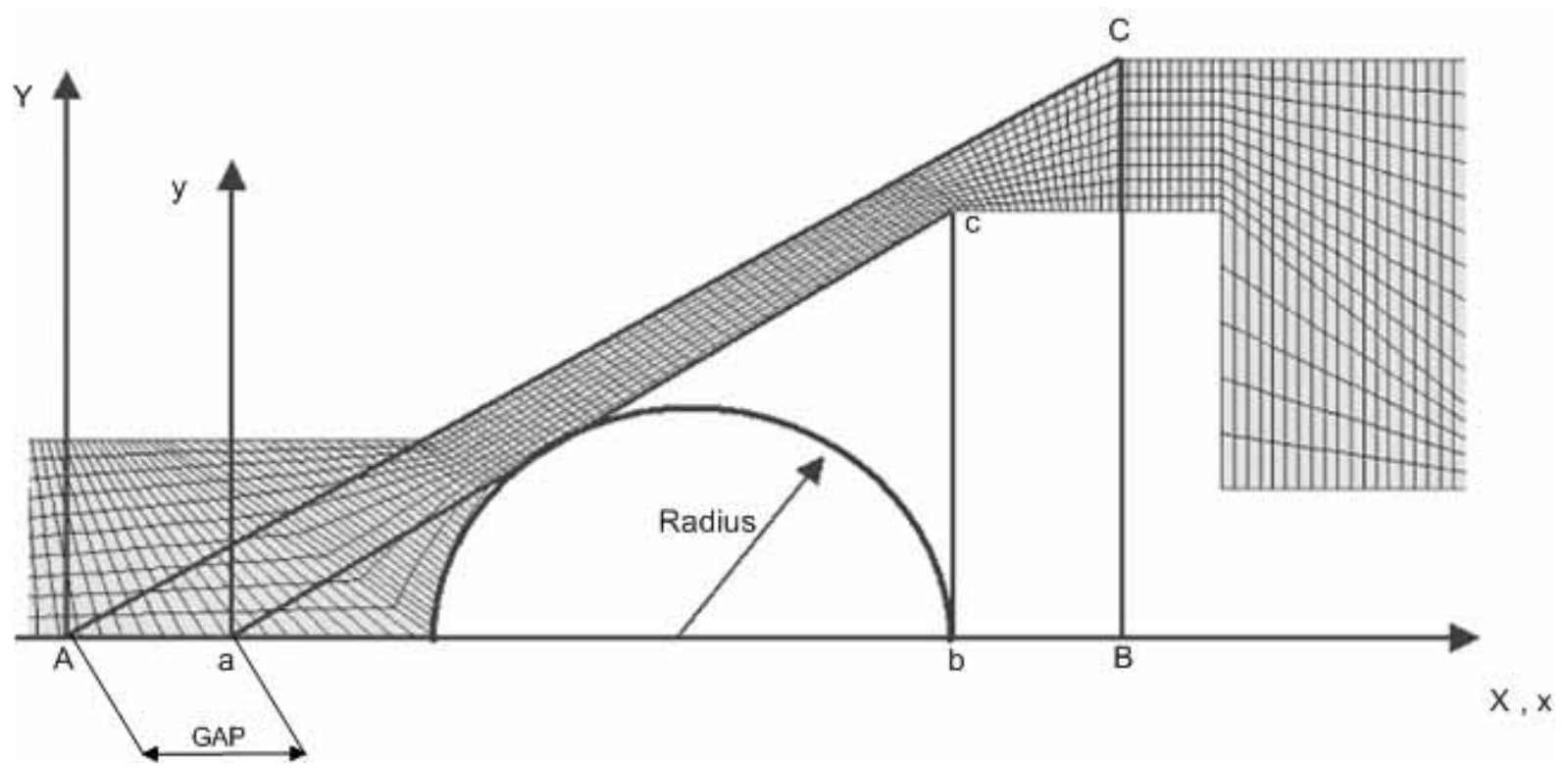

Fig. 3. Valve geometry and mesh refinement.

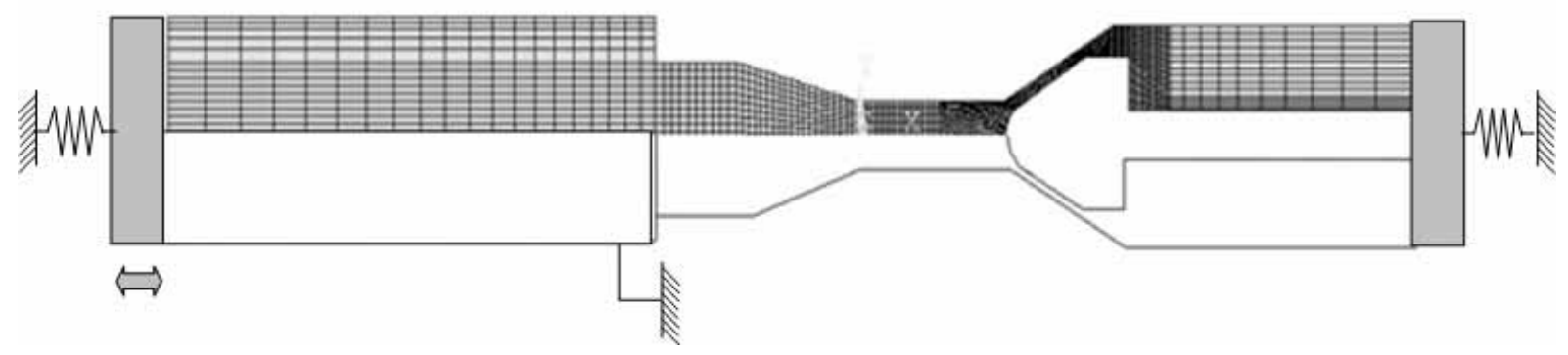

Fig. 4. Complete fluid-structure finite element model.

GAP and excitation frequency values combinations. The right bellows equivalent mass is attached to a spring with the same stiffness of the left bellows.

The problem was solved by using the Eulerian-Lagrangean algorithm [1], which formulation is made accordingly the flow chart of Fig. 5. The algorithm starts the time solution with an initial mesh. The fluid-structure interaction algorithm solves the equations on the fluid and on solid domains, independently of each other, and transfer the fluid forces and structure displacements across the fluid-solid interface. The mesh is updated and the algorithm continues to loop through the solid and fluid analyses until convergence of the force and the displacement variables are achieved for that time step or until the maximum number of stagger iterations is reached. After convergence the simulation proceeds to the next time step.

In this class of problems, it was verified that the convergence is highly dependent on the value of the relation between bulk modulus and the viscosity of the fluid. Larger values of this relation requires smaller time steps to guarantee the algorithm convergence. Three different values were investigated for the bulk modulus: $8.42 \times 10^{7}$ (A), $8.42 \times 10^{6}$ (B) and $5.9782 \times 10^{6}$ (C) which require a time step of $0.0005,0.01$ and 0.01 seconds, respectively, to guarantee the numeric convergence of the simulation.

Simulation results will be shown and compared with the experimental results in the next section. A total of 24 numeric simulations were made with GAP values equal to $0.06,0.1,0.15,0.225,0.35,0.5,0.75$ and $1.00 \mathrm{~mm}$, and with the three bulk modulus values. 


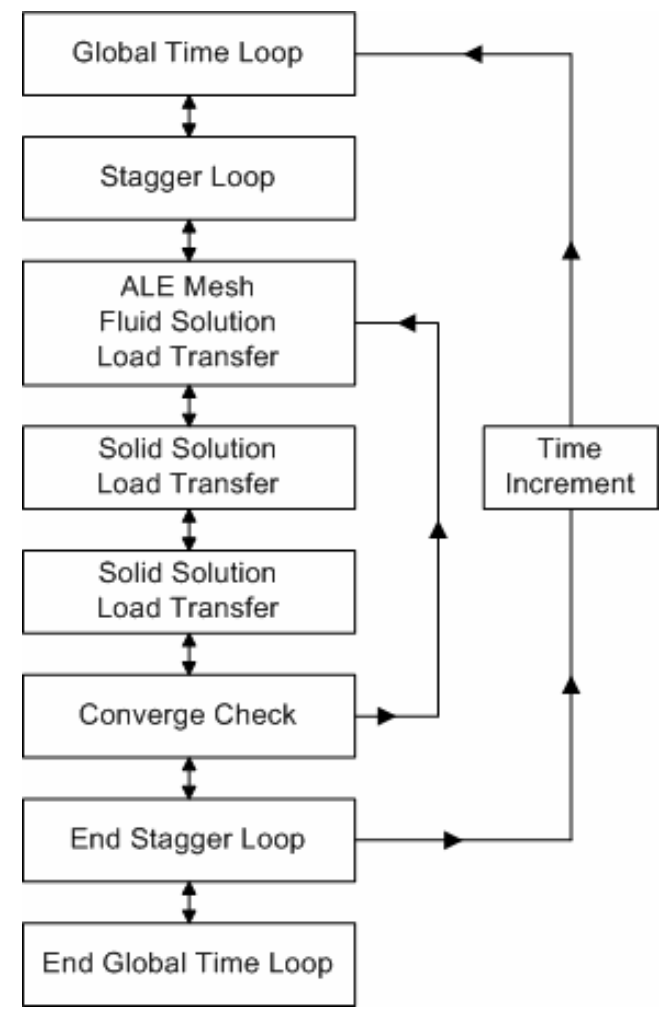

Fig. 5. Lagrangean-Eulerian algorithm.

\section{Experiments with the active damper device}

The active damper prototype designed by the finite element model was manufactured according the drawing presented by Fig. 6 .

To operate properly, the damper device must be filled with oil and the air bubbles must be removed to avoid bulk modulus variation with the changes of the dynamic pressure inside the valve. To achieve this task, the damper was filled with oil using a parallel system composed by a vacuum pump, two reservoirs and two manual valves, as illustrated in Fig. 7. A static pressure sensor measures the imposed internal pressure equal to 0.3 MPa. After verifying that there was no leak and that the static pressure was steady, the oil reservoirs were uncoupled, and the damper was ready to operate.

Some experiments were done to validate the finite element model of the damper, operating as a passive device. Harmonic forces at 5, 15 and $30 \mathrm{~Hz}$ produced by an electrodynamic shaker excited the device left bellows. In these tests, the damping force and the resulting velocity were measured for different GAP values. The force and velocity signals were captured by a computer based acquisition system. For these tests, the GAP position was set by an millimetric screw and measured by a dial gage.

The Fig. 8 shows the experimental set up, with the shaker coupled to the flexible bellows. A piezoelectric force sensor and an accelerometer measure, respectively, the input signal applied to the damper and its output. The velocity at the bellows was obtained by analog integration of the acceleration signal.

The results obtained for 20 investigated GAP values are shown in Fig. 9, for the tests where the excitation frequency is $30 \mathrm{~Hz}$. For each GAP value, the velocity and the damping force peak amplitudes were measured, resulting straight lines whose inclination is the damping coefficient. These results indicate that the damper has a linear behavior with the velocity.

The comparison between the simulated and experimental values of the damping coefficient is presented by Fig. 10. These results, obtained using an excitation frequency equal to $5 \mathrm{~Hz}$, shows that the damping coefficient increases 


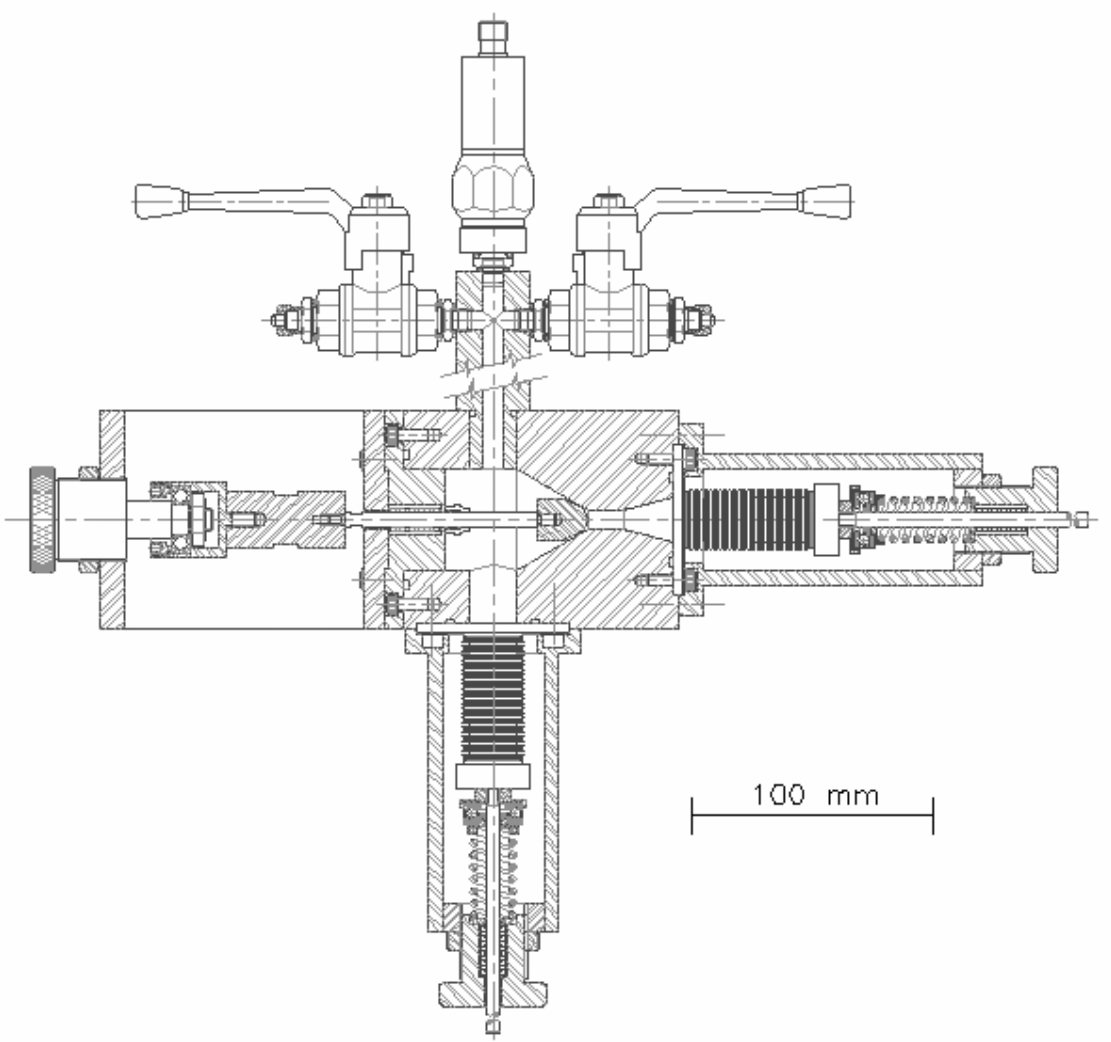

Fig. 6. Active damper prototype.

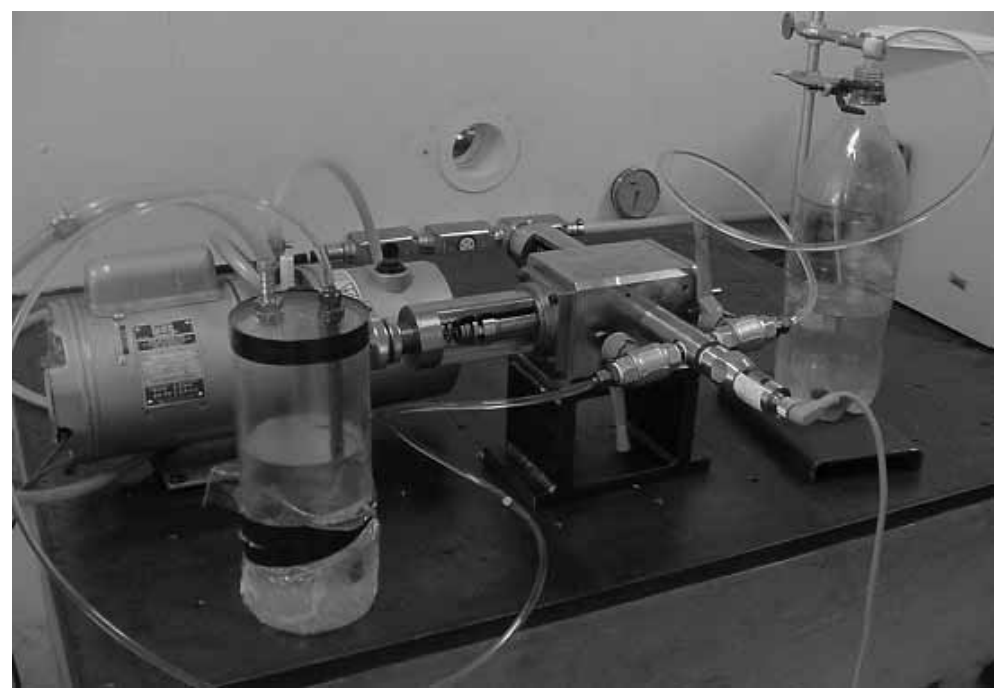

Fig. 7. Assembly of the active damper.

exponentially when the GAP decreases. The simulations were done with three different values of the ratio between the bulk modulus and the viscosity of the fluid. Small GAP values requires a finer mesh in the fluid region, and produces a larger finite element model. Therefore the computational effort is considerably increased. 


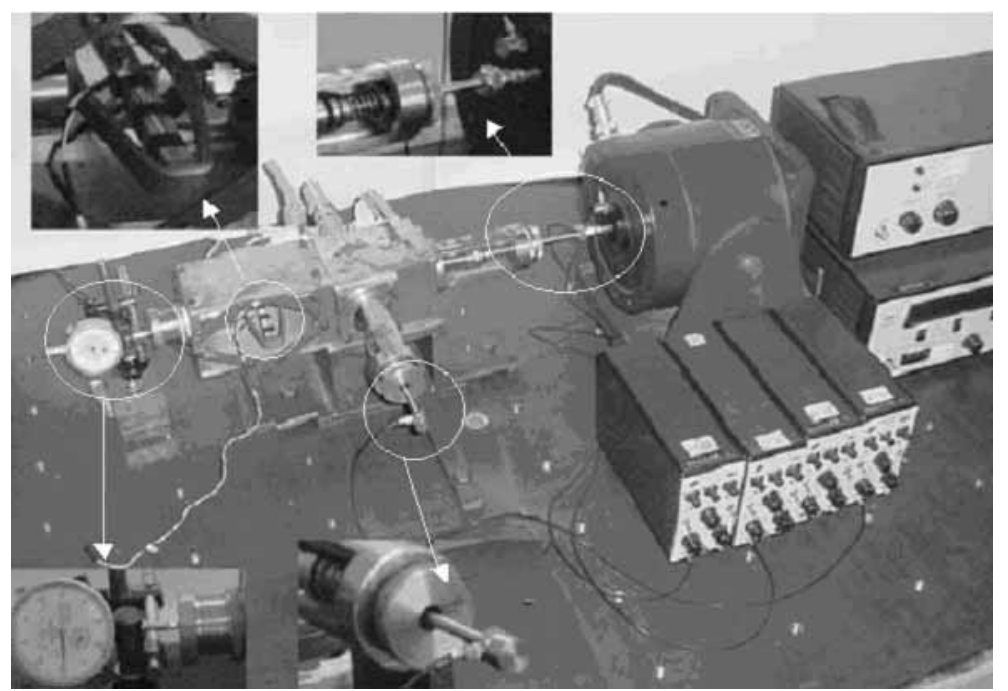

Fig. 8. Experimental set up of the active damper.

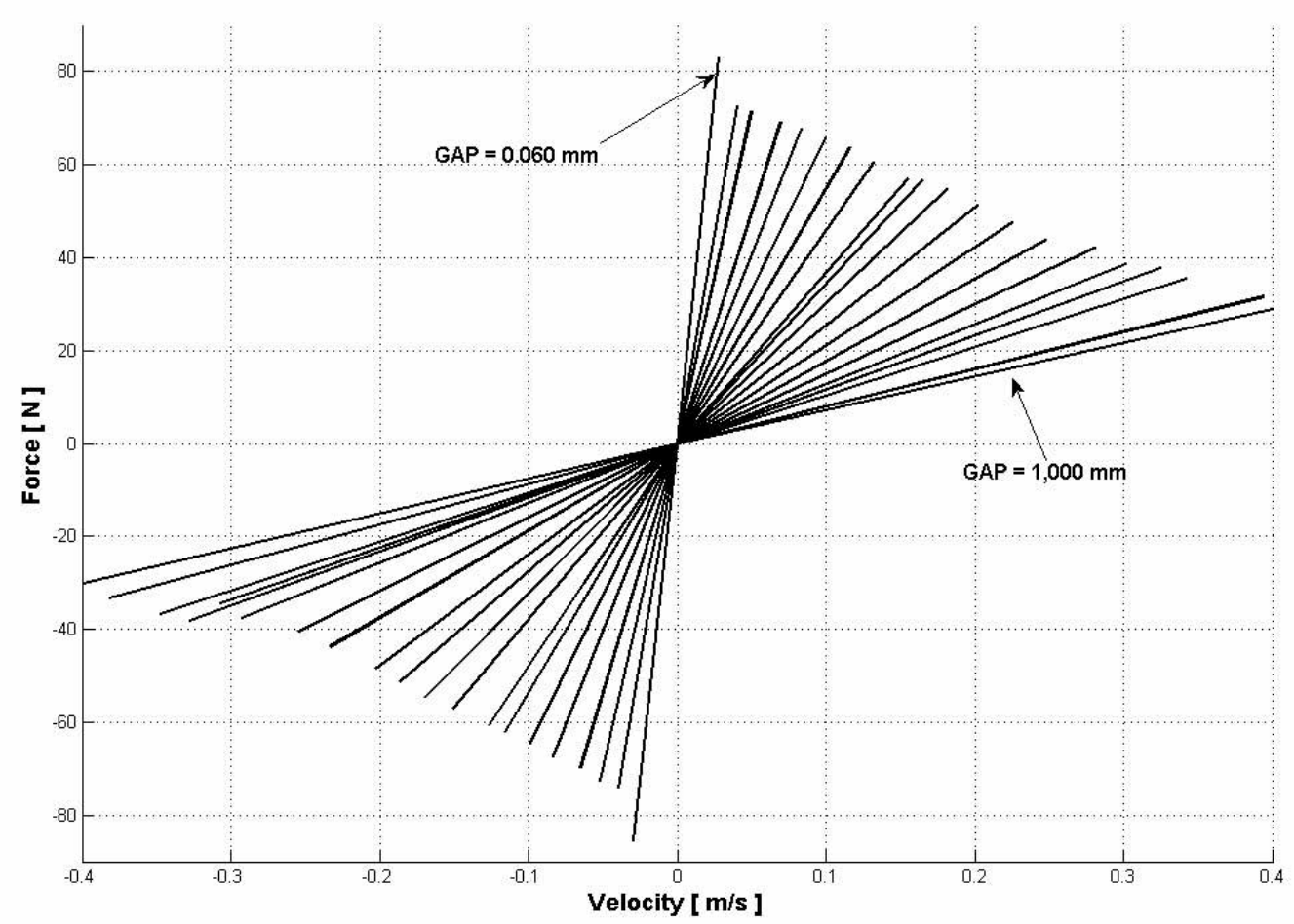

Fig. 9. Experimental relation between the damping force and the velocity for different GAP values.

Smaller ratio values (B and C) are unable to represent the damping coefficient for small GAP values. Better, but not perfect fitting is achieved for the larger ratio (A). As mentioned before, increasing the bulk modulus-viscosity ratio, the integration time step must be reduced and even so the numeric convergence is not always warranted. 


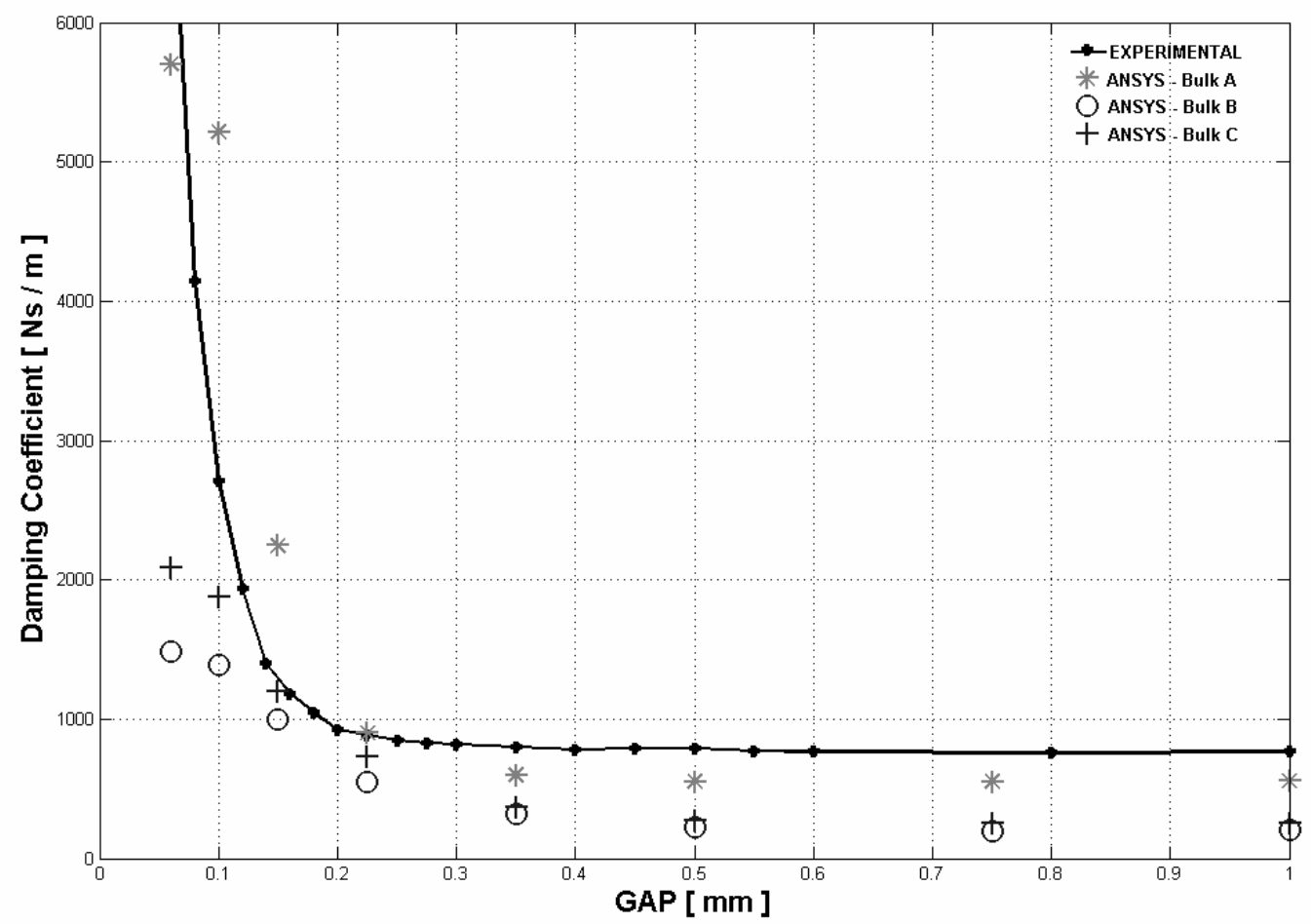

Fig. 10. Comparison between the simulated and experimental results for $5 \mathrm{~Hz}$ excitation.

As a consequence of these numerical requirements, the direct use of this model is not viable in real time control application.

Considering that the finite element model is validated by the experimental tests, it can be used to optimize the design of the damper, without the necessity to built new prototypes. The designer, with this model, will be able to study other valve configurations, different bellows characteristics, and so on.

A finite element model that represents the piezoelectric actuator was also developed but it was not coupled to the fluid-structure model due to the demand of high computational time and effort. At the present stage of the research, the actuator dynamics is not included in damper computational model. However, a reduced model of the damper, including the piezoelectric actuator was estimated from the frequency domain data obtained in experiments with the complete damper prototype. These results are shown in the next section.

\section{Active damper identification in the frequency domain}

The damper prototype was excited by narrow band random force applied by an electrodynamic shaker to the left bellows, in the frequency band from 0 to $100 \mathrm{~Hz}$. The experimental transfer functions represent the ratio between the acceleration and the force measured at the left bellows. The frequency response functions (FRF) shown by Fig. 11 demonstrate that the damper behavior can be modeled by a single degree of freedom vibratory system in this frequency band. The experiments were made for $20 \mathrm{GAP}$ values, spanning the displacement range of the piezoelectric actuator. For each GAP value, the physical parameters of the second order dynamic system were estimated. The difference of each second order system is the damping factor. In this same figure, the dashed line curves are the adjusted FRF used to estimate the properties of damper model. These estimates were obtained by the Nelder-Mead multidimensional non-linear optimization method. 

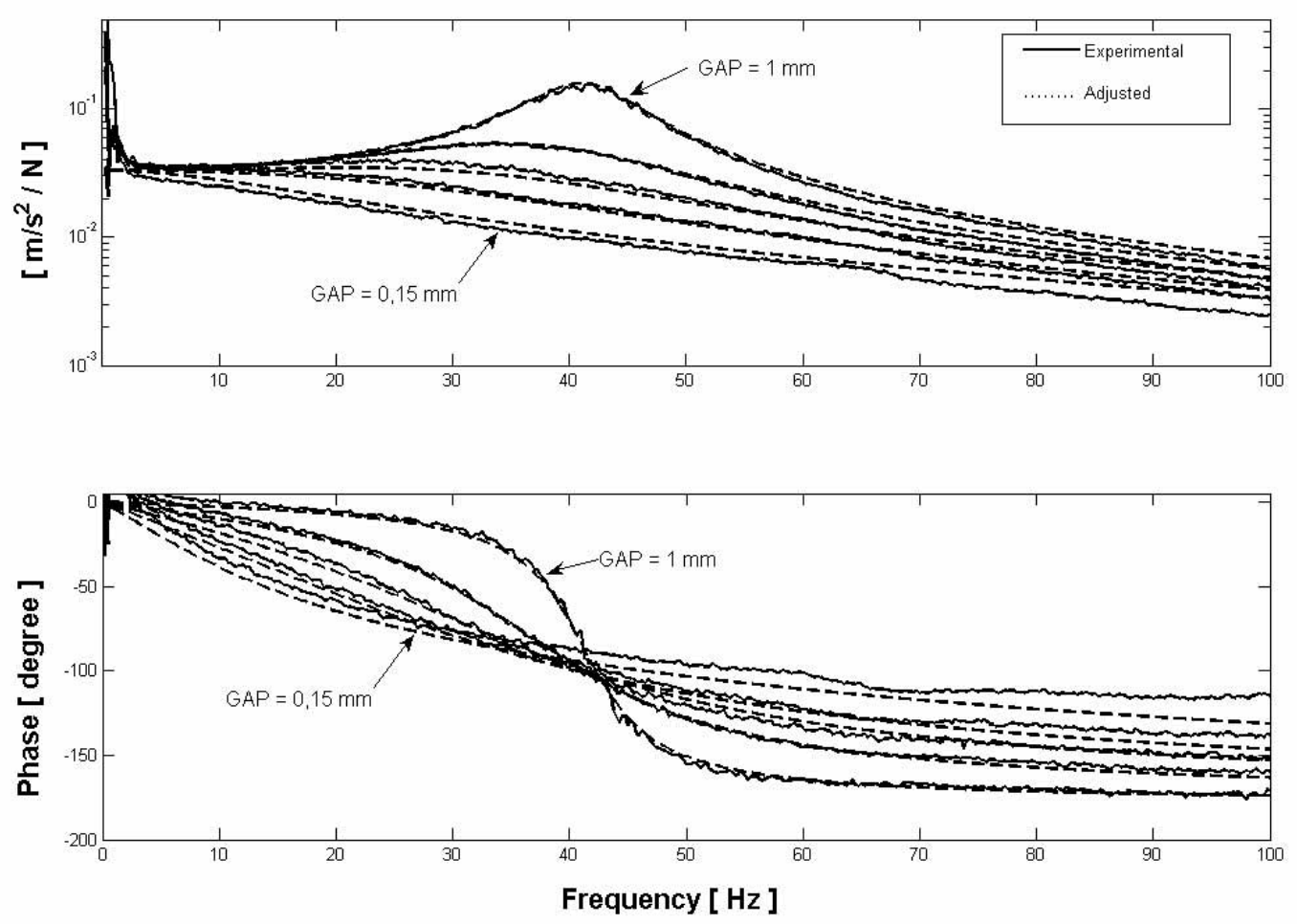

Fig. 11. Experimental and estimated FRF for different GAP values.

The damper generic mathematical model in the frequency domain is presented in the Eq. (1), where $K=$ $39.840 \mathrm{~N} / \mathrm{m}, \omega_{n}=38 \mathrm{~Hz}$ are, respectively, the estimated stiffness and the undamped natural frequency, $\xi$ is the variable damping coefficient, and $s=j \omega$.

$$
G(s)=\frac{1}{K} \cdot\left(\frac{\omega_{n}^{2}}{s^{2}+2 \xi \omega_{n} s+\omega_{n}^{2}}\right)
$$

Using the estimated FRF, the relation between the damping coefficient and GAP was calculated by an optimization technique, resulting the model described by Eq. (2).

$$
\xi=3.8252 \cdot e^{-6.2675 . G A P}+0.138 \cdot e^{-0.0046 . G A P}
$$

This model is used to simulate the damper device behavior in both passive (fixed GAP) and active (variable GAP) operation modes. The active system has a fuzzy control that generates the reference GAP to be imposed to the actuator, depending on the desired dynamic behavior of the system.

\section{Active damper with GAP fuzzy control}

The fuzzy logic treats the propositions with different degrees of pertinence of true or false values, in opposition to the classic logic, where the propositions assume only two possible values: false (0) or true (1). Teixeira [8] made an overview of the fuzzy sets theory and control. The fuzzy controller has a rules base on if-then that relates the linguistic variables of the input to the output, both represented by pertinence functions.

The first step of the fuzzy controller design is the definition of the physical input and output variables. In this application, the input variables are the displacement and the velocity of the damper, and the output is the GAP value. 
Table 1

Rules base of fuzzy controller

\begin{tabular}{cccc}
\hline V D & N & Z & P \\
\hline N & Small* $^{*}$ & Small & Small \\
Z & Medium & Large & Medium \\
P & Small & Small & Small \\
\hline
\end{tabular}
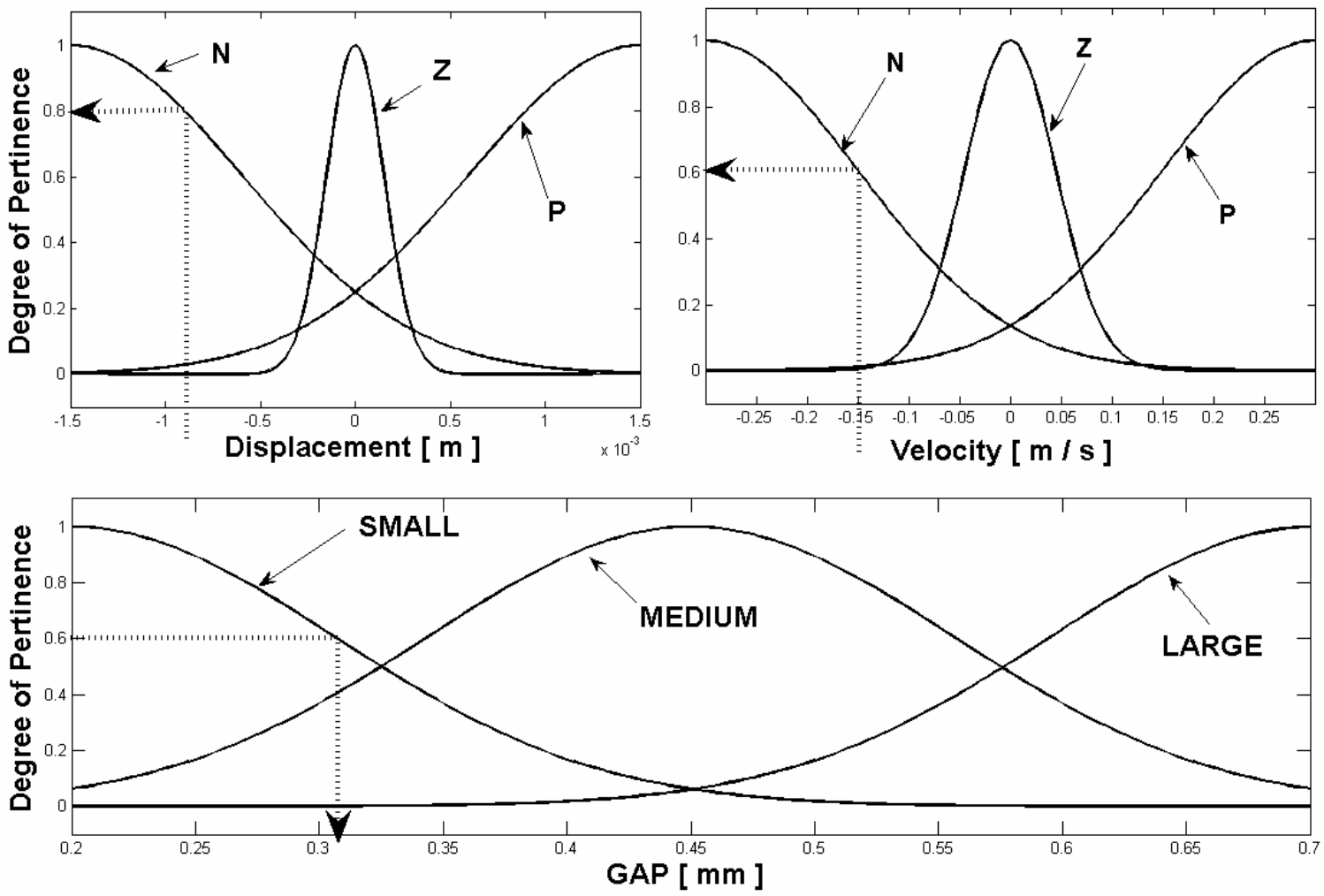

Fig. 12. Inputs and output pertinence functions of fuzzy controller.

The fuzzy controller can be capable to estimate the best GAP to be imposed in order to reach an specified performance index. In this application it is the damping factor required by the system. Each input and output variables were partitioned by 5 gaussian pertinence functions, as illustrated in the Fig. 12. The rules base is considered as the last stage of fuzzy controller design and it was obtained by the designer intuition. The adopted rules base is shown in Table 1.

To understand the Mamdami fuzzy algorithm [8] considers the rule marked with * on Table 1: If the displacement is Negative and the velocity is Negative, then GAP will be Small. Suppose the input displacement $\left(i_{1}\right)$ is about $0.8 \mathrm{~mm}$ and the input velocity $\left(i_{2}\right)$ is about $0.15 \mathrm{~m} / \mathrm{s}$. The Fig. 12 shows these values crossing the pertinence functions in 0.8 and 0.6 , respectively. Since the adopted inference method is the minimum between the degrees of pertinence of the two inputs, then 0.6 is the result of the fuzzification. This value of 0.6 crosses the small GAP pertinence curve resulting the output $\left(o_{1}\right)$, which corresponds to GAP equal to $0.31 \mathrm{~mm}$.

Using this fuzzy controller design and the second order damper model proposed in Section 4, numerical simulations were done to evaluate the active damper behavior. Both passive and active systems were submitted to a rectangular $300 \mathrm{~N}$ impulsive force with duration of $10 \mathrm{~ms}$.

On the passive system simulations the GAP was set to $0.45 \mathrm{~mm}$, which is equivalent to a damping factor equal to 0.3656 . On the active system simulations the fuzzy controller updates the GAP at each sampling interval, which is $d t=1 \mathrm{~ms}$. Therefore, the active system transfer function is modified at each sampling time. The displacement 

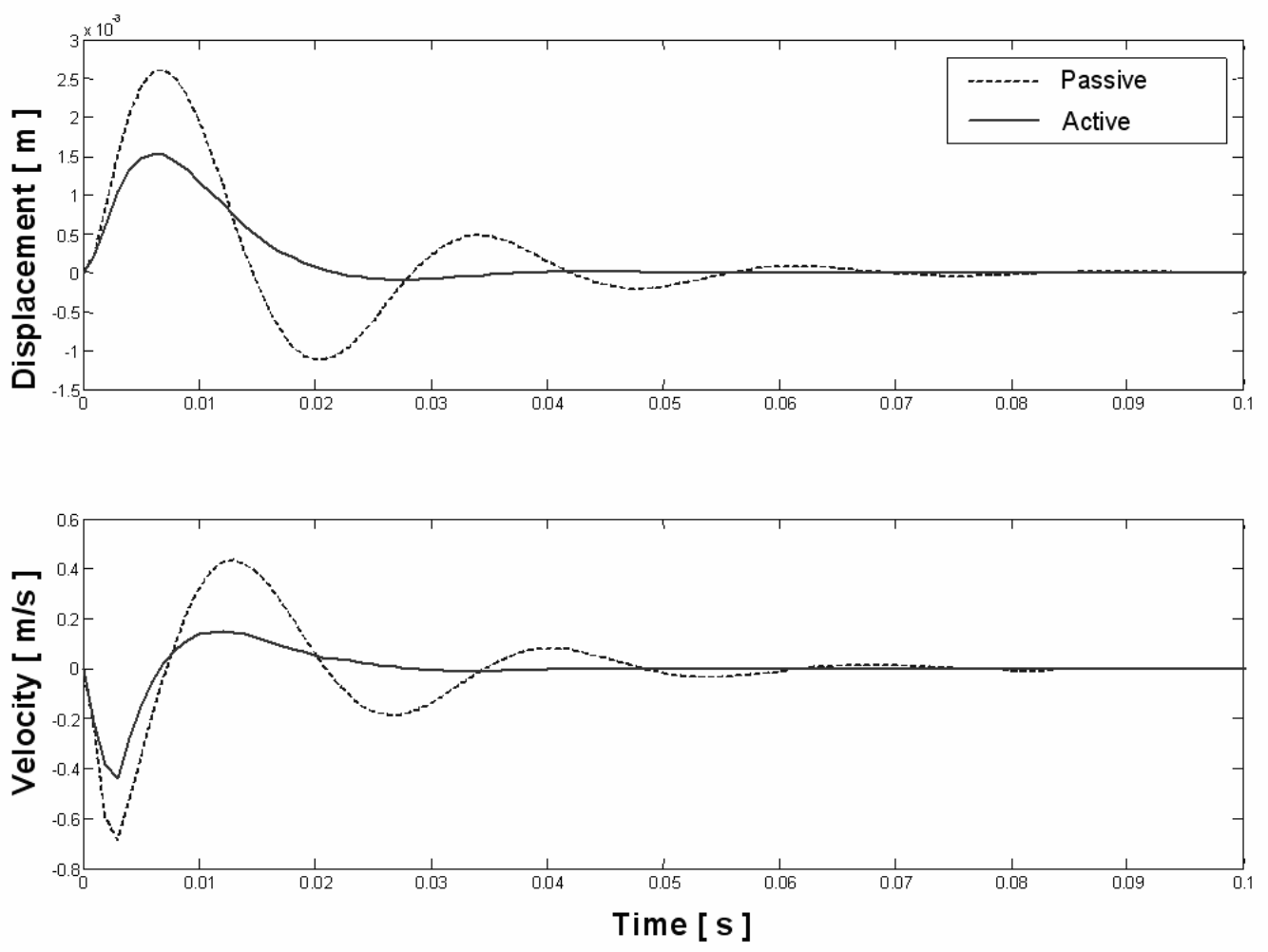

Fig. 13. Impulsive force response of the active and passive system.

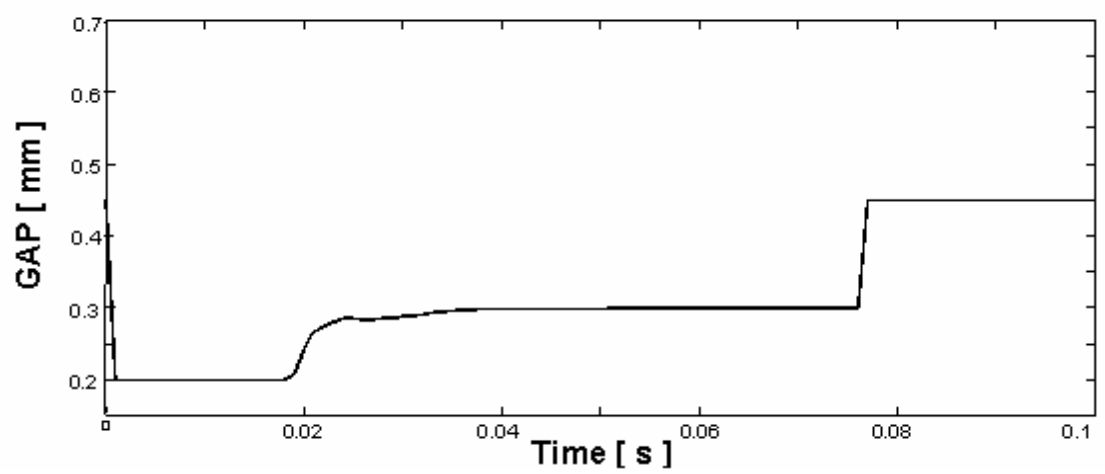

Fig. 14. Gap imposed by the fuzzy controller.

and velocity time domain results for the passive and the active system are presented by Fig. 13. The maximum displacement value of the active system resulted 1.8 times smaller than that of the passive case.

The GAP behavior, shown by Fig. 14, is the value inferred by the fuzzy controller in the simulations of the active system. In other words, the piezoelectric actuator needs to be supplied by a voltage that sets these valve core positions. As can be see in Fig. 14, in the instant of the impact the fuzzy controller close the valve generating the maximum damping factor and then the valve assumes a medium damping factor and finally according the performance index the valve return to its initial position. 


\section{Conclusions}

This paper presented two models of an active viscous damper and their experimental validation. The finite element model represents the device geometry and includes the fluid-structure interaction. This model was useful to analyze different configurations of the device and their associated steady and transient responses. The use of the simplified representation of the flexible bellows by a stiffness and an equivalent mass resulted a simpler computational model and proved to be effective to represent the device experimental behavior. Small GAP values demand a finer mesh at the region around the valve core and the use of higher bulk modulus values provides better adjustment of the damping force at low GAP values. It should be noted that the computational results are highly sensitive to the ratio between the fluid bulk modulus and the viscosity and also depend on the mesh refinement of the fluid domain.

The experimental tests carried out with the damper prototype in the time domain validate the finite element model. The experimental and simulation results show that the damping coefficient has exponential behavior with GAP and depends linearly on the velocity. The damper dynamics resulted to be very sensitive to small changes of the GAP value. Therefore, a piezoelectric actuator, which has a limited stroke range, can be used to control the valve orifice providing a large range of the damping coefficient.

However, for the control system design direct use of the finite element model is not viable due to the high computational processing time. To solve this task a reduced model was proposed to represent the active damper model. This model was adjusted by an optimization process using the prototype experimental results obtained in the frequency domain, by an excitation in the $0-100 \mathrm{~Hz}$ band. The model can be represented by a single degree of freedom vibratory system at each GAP value, which is related to a different damping coefficient. A fuzzy controller was designed to estimate the ideal GAP value that minimizes the vibration amplitude. Simulations were done to compare the passive and active system time response. The performance obtained for the active system resulted 1.8 times better than the passive, when an impulsive force excites the systems.

The coupling of the piezoelectric actuator finite element model to the damper fluid-structure model was not completed. At present date, a PID controller dedicated to command the actuator position and a special power amplifier to drive the low impedance piezoelectric pack are being designed. Then, the fuzzy control strategies will be used to generate the GAP reference to the input of the PID. After the completion of these tasks the complete model of the damper installed on a dynamic system will be experimentally verified.

\section{Acknowledgement}

The authors thank the financial support from CAPES, CNPq and FAPEMIG, Brazilian agencies.

\section{References}

[1] J. Donea, A. Huerta, J.-Ph. Ponthot and A.R. Ferran, "Chapter 14: Arbitrary Lagrangian - Eulerian Methods", in: Encyclopedia of Computational Mechanics, (Vol. 1), E. Stein, R. de Borst and T.J.R. Hughes, eds, Fundamentals. John Wiley \& Sons, Ltd. ISBN: 0-47084699-2, 2004.

[2] C.L. Giliomee and P.S. Els, Semi-active hydro pneumatic spring and damper system, Journal of Terramechanics 35 (1998), $109-117$.

[3] Hagopian, J. Der, L. Gaudiller and Maillard, Hierarchical control of hydraulic active suspensions of a fast all-terrain military vehicle, Journal of Sound and Vibration 222(5) (1999), 723-752. Article No. 1998.2082, available on line at http://www.ideallibraty.com.

[4] H.-U. Oh and J. Onoda, An experimental study of a semi active magneto-rheological fluid variable damper for vibration suppression of truss structures, Institute of physics publishing: Smart Materials and Structures 11, available online at stacks.iop.org/SMS/11/156, 2002, $156-162$.

[5] K.J. Kitching, K.J. Cole and D. Cebon, Performance of a semi-active damper for heavy vehicles, submitted to ASME Journal of Dynamic System Measurement and Control, June 1998.

[6] B.E. Launder and D.B. Spalding, The numerical computation of turbulent flows, Computer Methods in Applied Mechanics and Engineering 3 (1974), 269-289.

[7] R.L. Teixeira, F.P. Lépore and J.F. Ribeiro, Active Damper System Design and Control - Part A and B, COBEM 2003: 17O Congresso Internacional de Engenharia Mecânica - São Paulo - Brasil, 2003.

[8] R.L. Teixeira, Uma metodologia de projeto de controladores híbridos inteligentes com aplicações no controle ativo de vibrações mecânicas, Dissertação de Mestrado, Universidade Federal de Uberlândia - MG, 2001. 

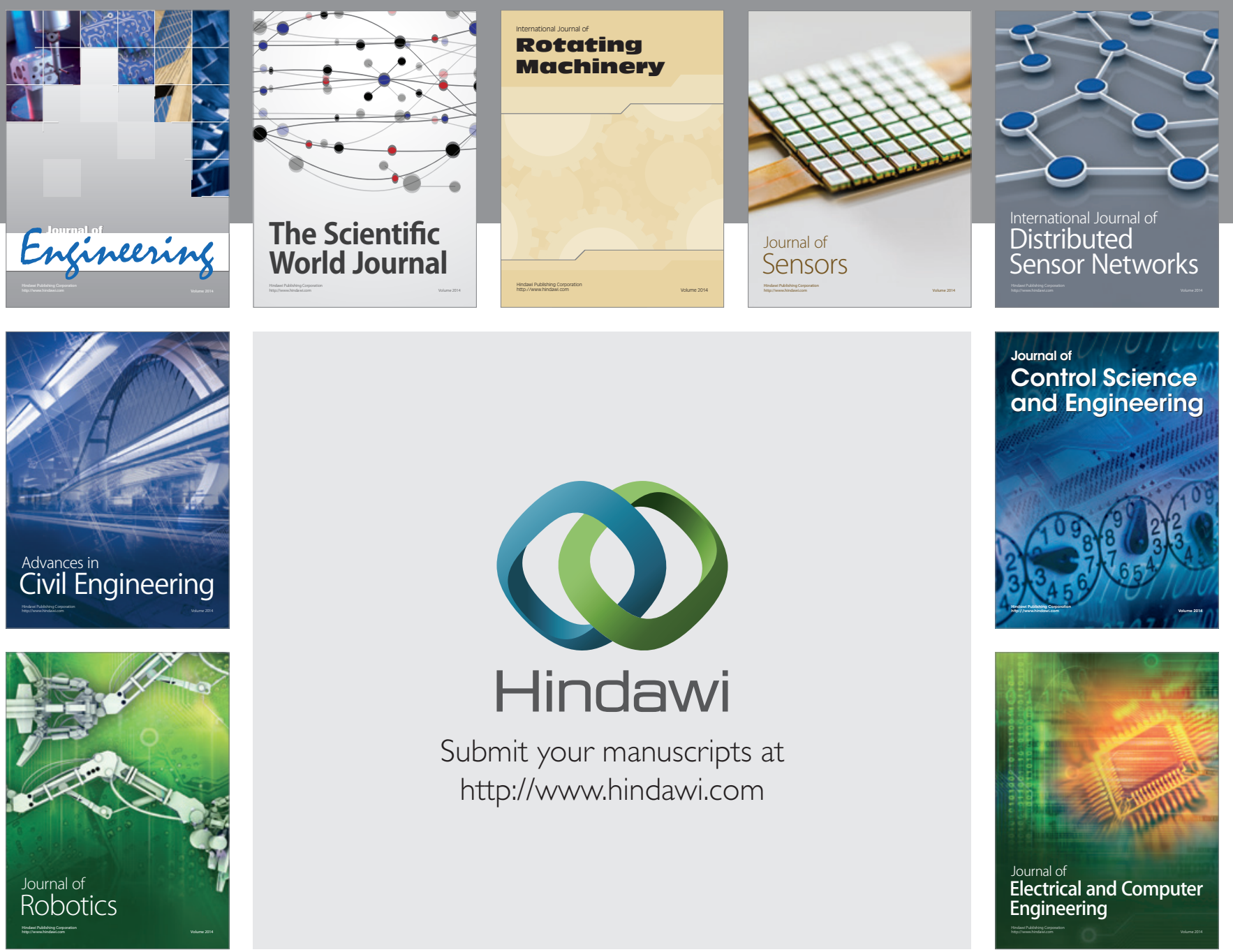

Submit your manuscripts at

http://www.hindawi.com
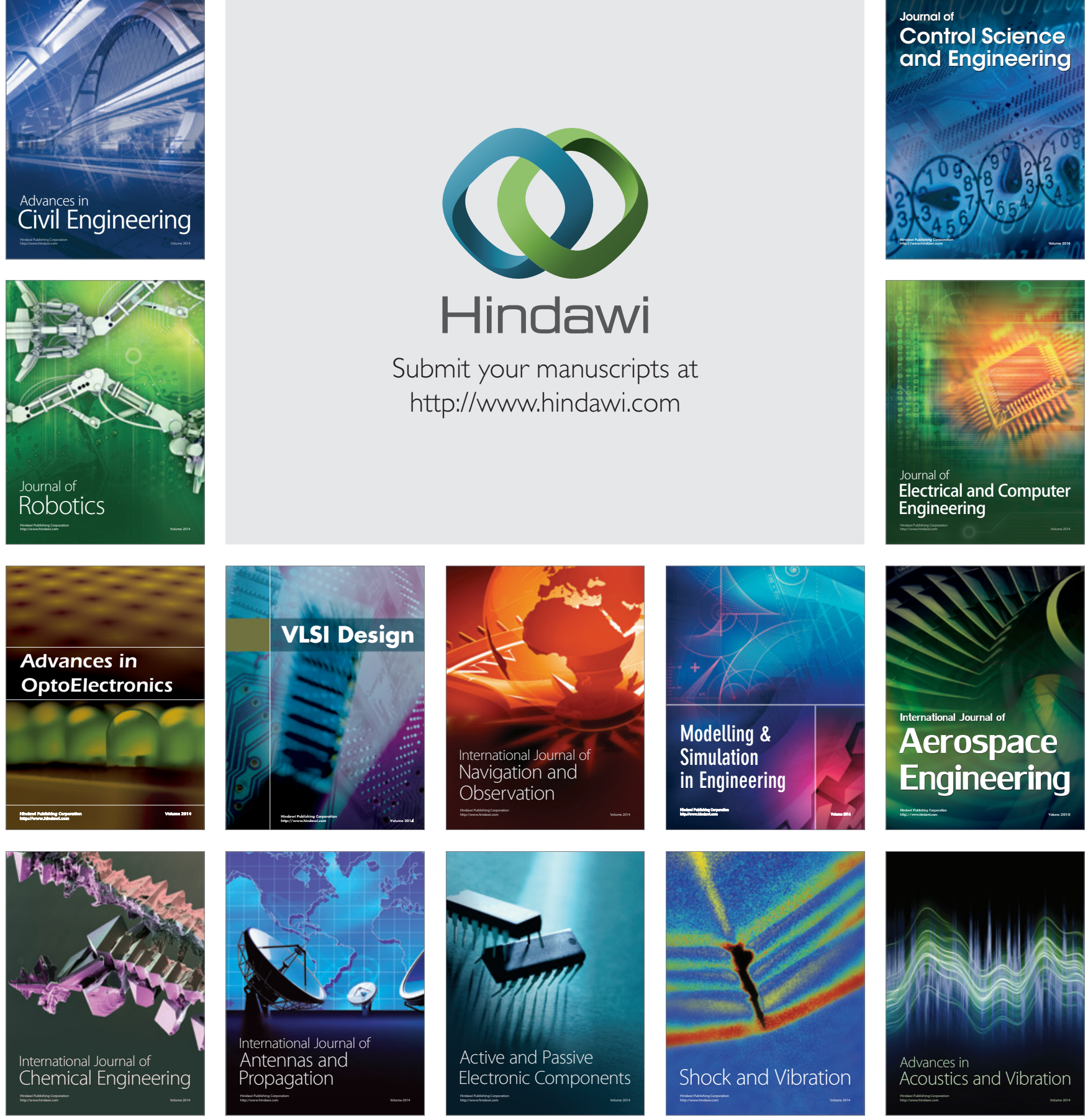